\title{
Methods for the Geographic Representation of Interpersonal Relationships and Social Life
}

\author{
Clio Andris ${ }^{\mathrm{a}, *}$, Dipto Sarkar ${ }^{\mathrm{b}}$ \\ ${ }^{a}$ School of City + Regional Planning and School of Interactive Computing, Georgia Institute of Technology, clio.andris@gmail.com \\ ${ }^{b}$ Department of Geography, National University of Singapore, dipto.sarkar@nus.edu.sg \\ * Corresponding author
}

Keywords: Social Flows, Social Networks, Personal Relationships, Friends, Human Behaviour

Interpersonal relationships are an important part of social and personal health. Studies of social capital show that individuals and communities with stronger ties are have an economic and health advantage. Yet, loneliness and isolation are becoming major public health issues. There is a pressing need to measure where relationships are strong and how accessible one's social ties are, in order to learn how to better support face-to-face meetings and promote social health in society. However, the datasets we use to study people and human behaviour are most often mobility data and census data-which tell us little about personal relationships. These data can be augmented with information about where people have ties, and how their relationships unfold over geographic space. The data we use to study the built environment include building footprints and infrastructure, and we can annotate these data by how (well) infrastructure supports different kinds of relationships, in order to ask new questions about how the landscape encourages relationships.

We suggest a list of methods for representing interpersonal relationships and social life at various socio-spatial levels of aggregation. We give an example of each, with an effort to span various use cases and spatial scales of data modelling.

Dyads (line) and Ego-based (star): This geometric model represents a relationship between two individuals (Figure 1A). The individuals can be geolocated to households, administrative units, real-time locations, etc. The tie can be given a nominal category such as family or co-worker, and edge weights that signify reported relationship strength, frequency of contact, frequency of face-to-face meeting, et cetera. Star models represent a central individual and his/her geolocated ties (that radiate from the centre). The star illustrates the theoretical concept of personal extensibility. ${ }^{1}$

Points of Interest (points): Points of interest provide a place-based perspective (note that these entities can also be represented as polygons such as building footprints, or lines such as gradients of interaction on a subway ${ }^{2}$ ). Certain places are better suited for fostering relationships than others (Figure 1B), and each can be annotated with their ability to foster: new ties (a nightclub), gender-bonding ties (bowling leagues), romantic ties (romantic restaurants), inter-generational ties (a religious facility), professional ties (conferences), et cetera.

Polygons/Administrative Units (polygons): These data are attached to administrative areal units (Census boundaries, provinces, zones, etc.). The data represent surveyed data on relationship-related variables in censuses, social surveys and social capital surveys. These surveys ask about trust, friendliness with neighbours, social life, belongingness to institutions, and more (Figure 1C), illustrating the social health of an area.

Aggregate Flows and Social Networks (lies and networks): This model illustrates the geolocated, social ties within a spatial extent, i.e. the social networks of a group of many people over a large extent (Figure 1D). Data can be sourced from social media, telecommunications patterns, and other declarations of relationships.

Regions (polygons): Regions, that may describe neighbourhoods within one city, or an agglomeration of cities, can be defined by social ties. Instead of commuting or economic ties, regions are defined by a preponderance of social ties within a given polygon, and a lack of ties between polygons (or between the polygon and any external area). Social regions represent a likeness and strong ties between the people that live within the region (Figure 1E).

Given these methods for representing social life and interpersonal relationships as GIS data, new questions may arise. At the dyadic level: how can we map the presence of a relationship between two people? At the ego-based level: how far and with what kind of diversity do people have ties? At the point of interest level: what kinds of mapable data can describe places' ability to create new relationships and foster existing relationships? At the polygonal level: what kinds of mapable data can show where relationships are strong or weak? At the levels of flows and networks: what kinds of mapable data can describe systems of diffusion? At the regional level: what physical and administrative boundaries guide social ties?

For cartographers and geographic modellers looking to study social life, data acquisition, analysis, and mapping are challenges. The point of this extended abstract is to inventory the possibilities of mapping these data, open a dialog for

\footnotetext{
${ }^{1}$ See: Adams, P. C. (1995). A reconsideration of personal boundaries in space-time. Annals of the Association of American Geographers, 85, 267-285.

${ }^{2}$ Swyngedouw, E. (2013). The segregation of social interactions in the red line L-train in Chicago. Symbolic Interaction, 36, $293-$ 313.
} 
experimenting with what kinds of symbologies, associated variables, classification schemes, visualization techniques and data collection opportunities are available for this purpose. We also hope to create spaces for comparative studies that describe the implications of these choices. In our search, we find that the major research challenges are the following: 1) privacy 2) geolocatable data 3) qualitative vs. quantitative data and 4) assurance statistically-significant samples sizes 5) analysis and modelling 6) visualization. Nevertheless, our goal is to make these indicators and data more GIS-friendly and available to geospatial analysts, modellers and cartographers.

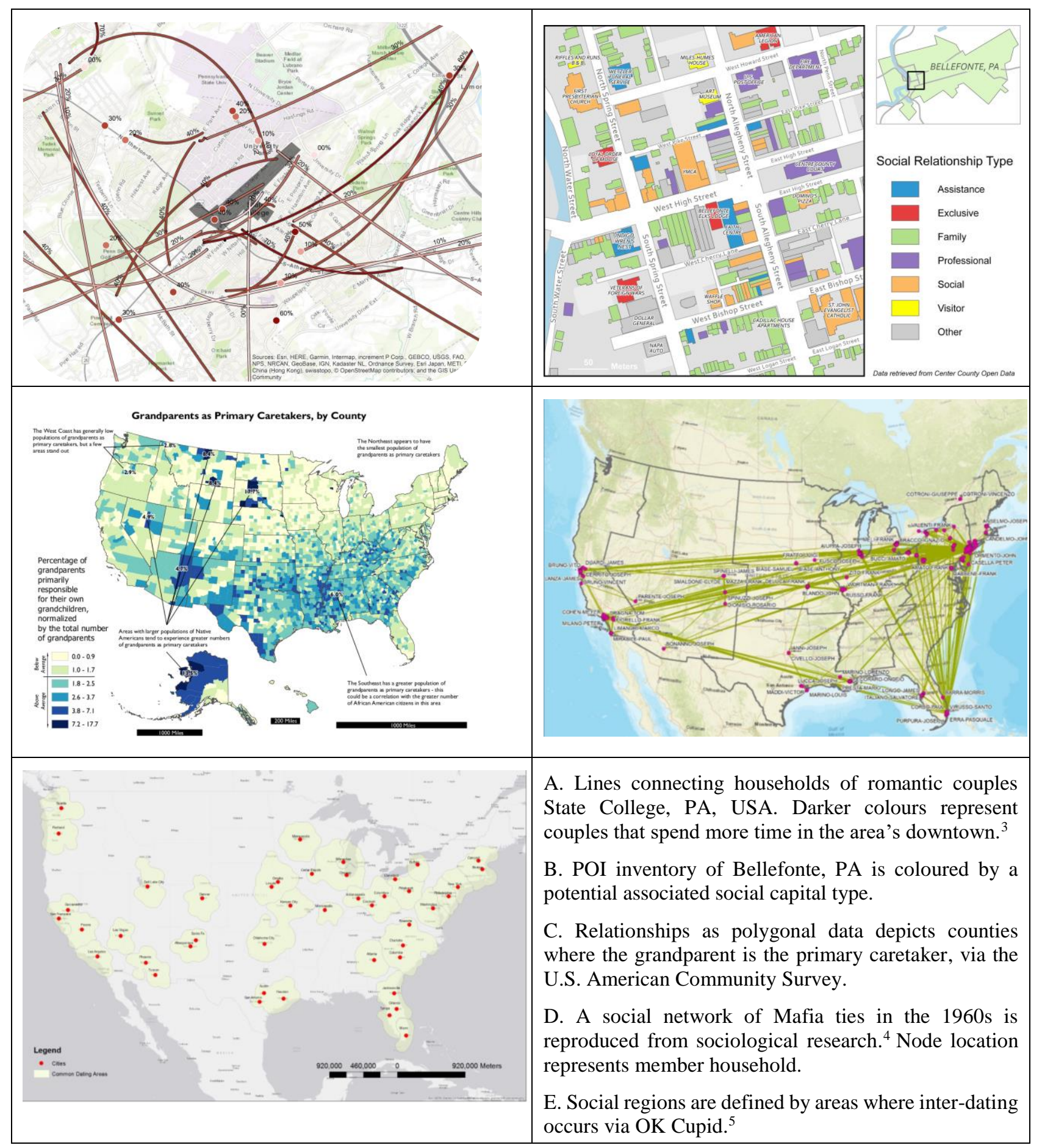

\section{Figure 1: Five visual examples of mapping interpersonal relationships and social life.}

\footnotetext{
${ }^{3}$ Penn State Friendly Cities Lab. Romantic relationships survey $(n=58)$ given in 2017. Dots represent couples that live together. ${ }^{4}$ DellaPosta, D. (2017). Network closure and integration in the mid-20th century American mafia. Social Networks, 51, 148-157.

${ }^{5}$ McMenamin, B. (2017). The Pitfalls of A/B Testing in Social Networks. OKCupid. https://tech.okcupid.com/the-pitfalls-of-a-btesting-in-social-networks/ 2017. Map by Michelle Burns.
} 